\title{
SIGNS
}

\section{The hilum overlay sign in a child}

\section{J van Wyk, MB ChB
N Mahomed, MB BCh, FCRad (Diag) (SA)
Department of Diagnostic Radiology, Chris Hani Baragwanath Hospital
and University of the Witwatersrand, Johannesburg}

\section{Case history}

A 9-month-old girl presented with a 1-month history of coughing. A chest X-ray (CXR) was performed, and the antero-posterior view demonstrated the hilum overlay sign. Furthermore, there was absence of the azygo-oesophageal line and bilateral paraspinal lines. The lateral CXR revealed a posterior mediastinal mass. Subsequently, a computed tomogram of the chest confirmed a posterior mediastinal mass, with calcifications and intraspinal extension consistent with neuroblastoma. Histological analysis confirmed this mass as a neuroblastoma.

\section{The sign}

Felson described the hilum overlay sign as the visualisation, on a frontal $\mathrm{X}$-ray, of the first bifurcation of either the left or right pulmonary artery more than $1 \mathrm{~cm}$ medial to the lateral edge of the cardiac shadow. This is highly suggestive of a mediastinal mass. ${ }^{1,2}$

The hilum overlay sign is present on a frontal chest radiograph when normal hilar structures can be visualised through a mass, which implies that the mass is either anterior or posterior to the hilum. ${ }^{3}$ The presence or disruption of mediastinal lines may further aid in the localisation of

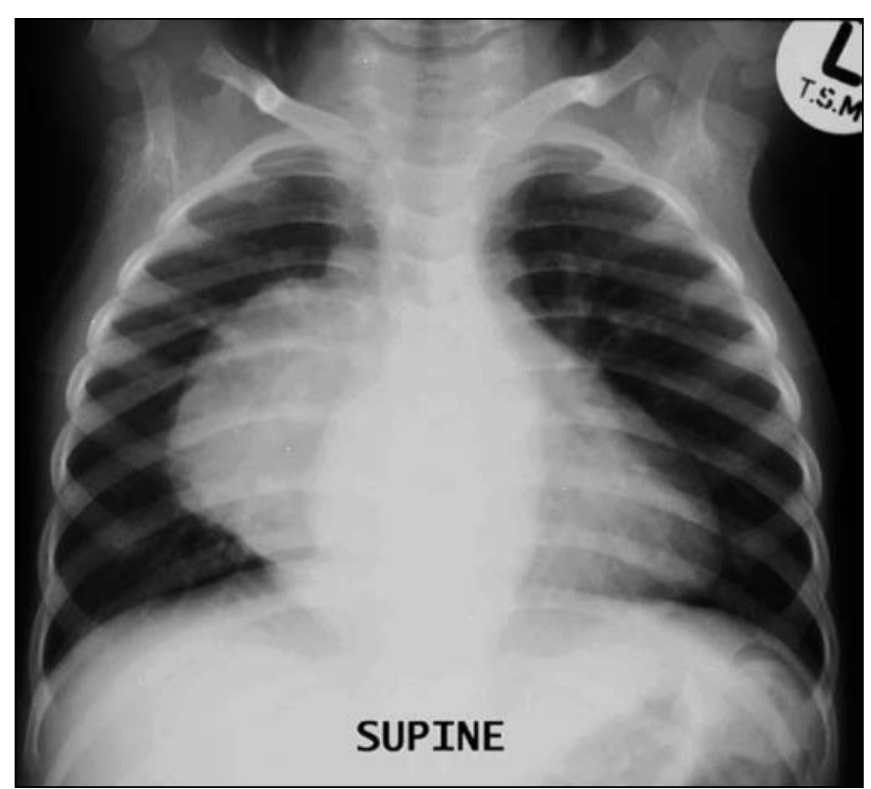

Fig. 1. AP CXR demonstrates the hilar overlay sign, with normal hilar structures visualised through the mass, and the absence of the azygooesophageal and bilateral paraspinal lines.

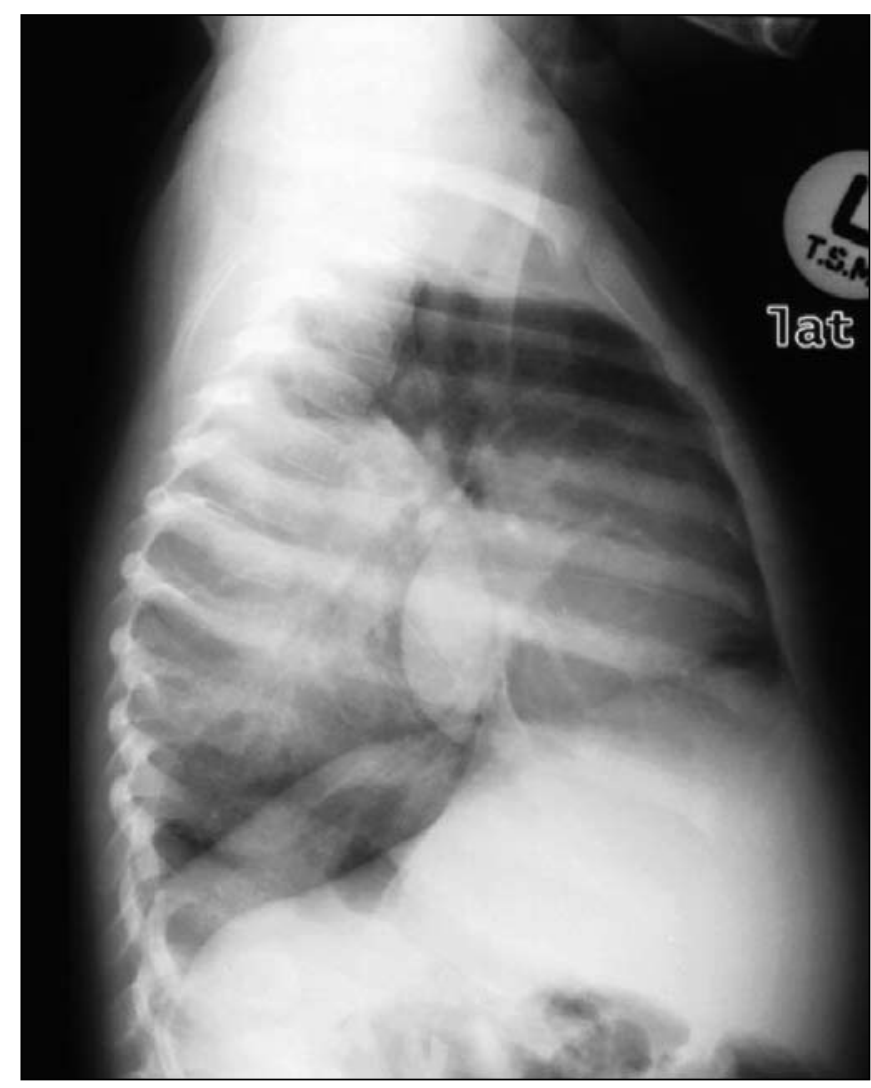

Fig. 2. Lateral CXR confirms a posterior mediastinal mass.

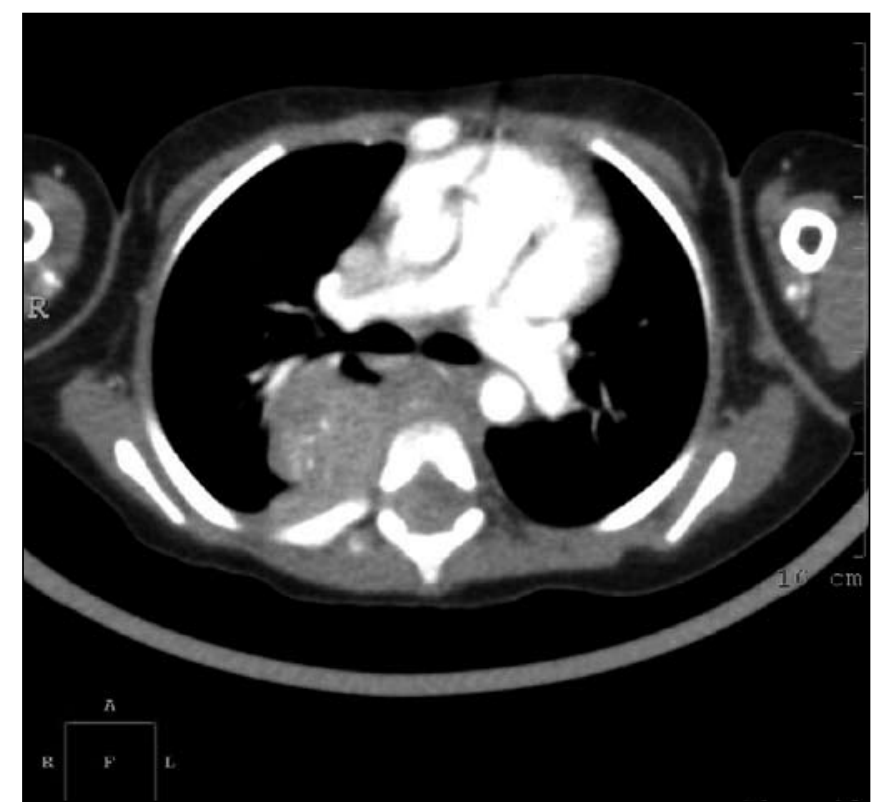

Fig. 3. Post-contrast axial chest CT at the level of the mediastinum demonstrates a poorly enhancing, predominantly right sided, posterior mediastinal mass with calcification and right neural foramen extension. 


\section{SIGNS}

the mass. The absence of the paraspinal and azygo-oesophageal lines suggests that a mass is located in the posterior mediastinum. The sign also enables us to distinguish a mediastinal mass from a prominent cardiac silhouette. ${ }^{4}$

A typical posterior mediastinal mass displaying the hilum overlay sign is neurogenic in origin in the majority $(88 \%)$ of cases. ${ }^{5}$ When there is calcification or adjacent bony erosion on a radiograph, it is highly suggestive of neuroblastoma. ${ }^{5,6}$ It may displace and invade adjacent structures and may even cross the midline. ${ }^{6}$ Most (70 - 90\%) neuroblastomas usually occur in the first 5 years of life. ${ }^{5,6}$
1. Felson B. The mediastinum. Semin Roentgenol 1969;4:41-58.

2. Felson B. More chest roentgen signs and how to teach them. Annual oration in memory of L Henry Garland, M.D., 1903-1966. Radiology 1968;90:429-441.

3. Whitten CR, Khan S, Munneke GJ, Grubnic S. A diagnostic approach to mediastinal abnormalities. RadioGraphics 2007;27:657-671.

4. Lesslie M, Chasen MH, Munden RF. Imaging of the mediastinum in oncology. Applied Radiology 2007;36(1): 8-19.

5. Merten DF. Diagnostic imaging of mediastinal masses in children. AJR 1992;158:825-832.

6. Strollo DC, Rosado-de-Christenson ML, Jett JR. Primary mediastinal tumors part II: Tumors of the middle and posterior mediastinum. Chest 1997;112:1344-1357.

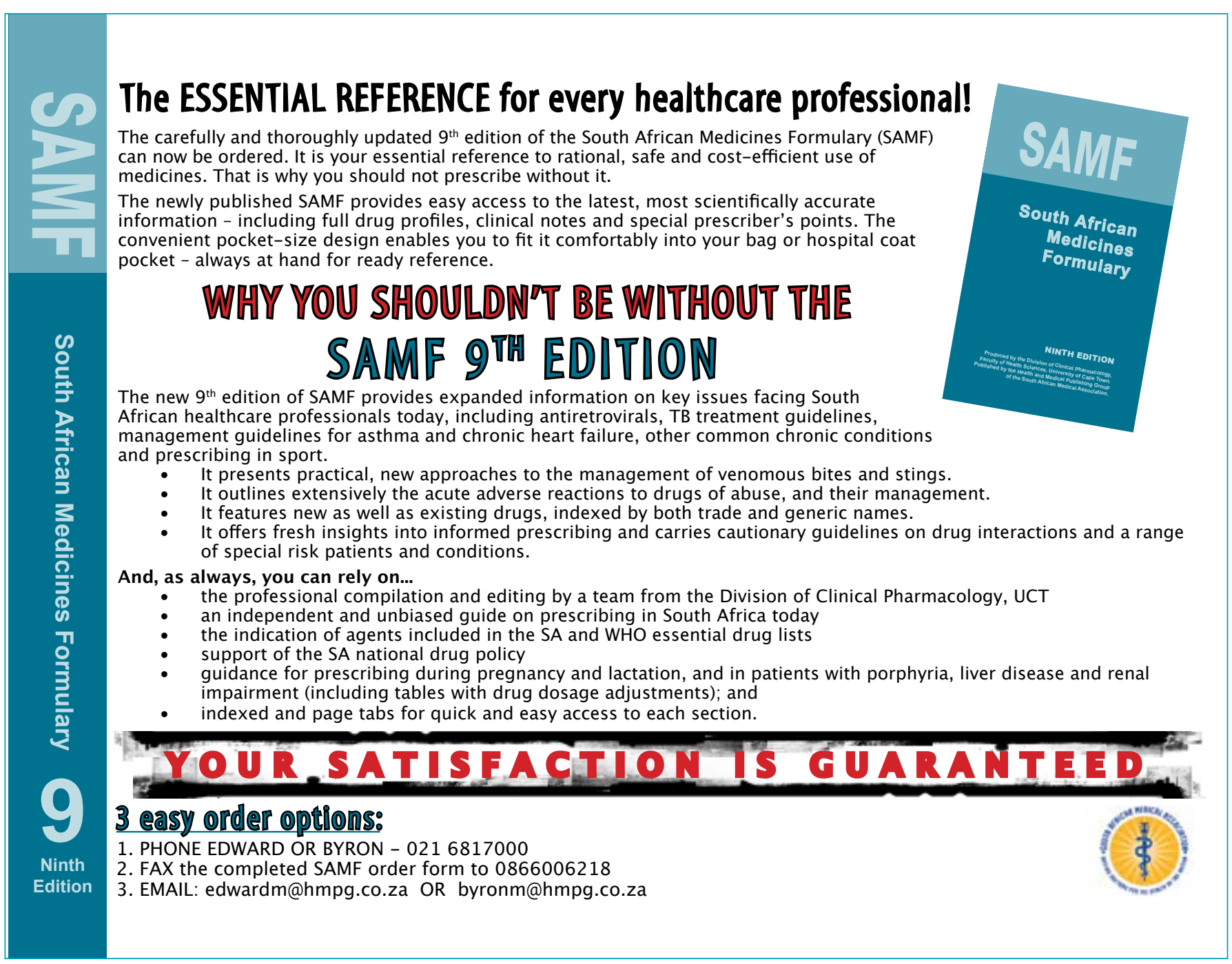

